\title{
Swiss Quality Award: et les gagnants sont... les patients!
}

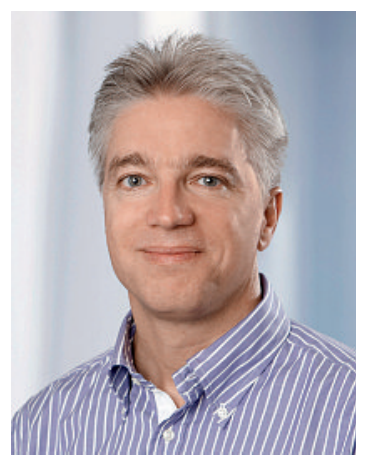

Cette année, lors de la quatrième édition du Symposium national pour la gestion de la qualité dans le secteur de la santé, quatre projets exceptionnels se sont vu remettre le Swiss Quality Award 2011 (www.swissqualityaward.ch). Notons toutefois que les 97 autres projets également en lice l'auraient tout aussi bien mérité. Cette cérémonie des oscars du domaine médical suisse a clairement mis en évidence la grande diversité et le niveau des initiatives qualité dans notre pays: des mesures de prévention à l'évolution des technologies de pointe pour blessés graves en soins intensifs, tous les domaines étaient représentés. Autant d'initiatives qui montrent la motivation profonde de leurs auteurs de vouloir améliorer la prise en charge et la sécurité des patients. Ce concours témoigne également de l'énergie déployée par les médecins pour améliorer la qualité des soins et pour faire connaître leurs activités allant dans ce sens. Une fois de plus, on a vu que ce sont les initiatives et les projets

\section{1 projets étaient en lice pour le Swiss Quality Award 2011.}

nés du contact quotidien avec les patients qui recèlent le plus fort potentiel de progrès véritable pour ces derniers. La FMH combat d'ailleurs vivement les pseudo-initiatives qualité dont le seul objectif est de satisfaire aux exigences d'un article de loi. De telles initiatives sont vaines et improductives.

Le concours pour la qualité des soins a également souligné l'importance que revêt la collaboration. En effet, la majorité des projets en lice sont le fruit d'un travail d'équipe.
Comme le dit bien mon adage favori «Si tu veux aller vite, vas-y seul; si tu veux aller loin, vas-y avec les autres», seul un travail d'équipe peut produire de tels résultats. Partisan convaincu de l'intelligence collective, je suis persuadé que l'échange interdisciplinaire est le seul moyen d'avancer au sein de ce milieu complexe qu'est le secteur de la santé. La place Suisse offre d'ailleurs des conditions idéales que nous devrions davantage mettre à profit. Bien des pays nous envient notre matière grise, nos hautes écoles reconnues, notre système politique stable et notre situation financière solide ou éprouvée. Tâchons de ne pas l'oublier lors des débats actuels sur la santé.

\section{Le concours récompense des projets novateurs - qui profitent avant tout aux patients.}

La remise du Swiss Quality Award 2011 a été pour nous une occasion de nous réjouir. Malgré un environnement toujours plus difficile et malgré les nombreuses discussions, pas toujours très positives, qui ont cours en politique de la santé, nous avons pu montrer à tous, notamment aux politiciens, que les médecins ont toujours autant de plaisir à exercer ce beau métier qui est le leur et que leur désir d'innover est intact. Lors de la remise des prix, Pascal Strupler, directeur de l'OFSP, mentionnait que de tels projets devraient inciter tous les acteurs de la santé à poursuivre leurs efforts dans le but d'améliorer le système de santé - des paroles qui, nous l'espérons, auront également trouvé un écho auprès du DFI et de l'OFSP. L'année prochaine, nous récompenserons à nouveau des projets exceptionnels, et nous montrerons à nouveau à tous notre engagement quotidien pour nos patients!

Dr Daniel Herren MHA, Membre du Comité central de la FMH, Responsable du domaine DDQ 